\title{
Total internal reflection holography for optical interconnections
}

\author{
Damien Prongué* \\ Hans Peter Herzig \\ University of Neuchâtel \\ Institute of Microtechnology \\ $\mathrm{CH}-2000$ Neuchâtel, Switzerland \\ E-mail: herzig@imt.unine.ch
}

\begin{abstract}
We applied near-field total internal reflection holography to solve the problems of miniaturization of lenslet arrays. A regular $100 \times 100$ lenslet array with a $390-\mu \mathrm{m}$ focal length and a nonregular lenslet array for clock distribution to a specially designed VLSI circuit were recorded in a planar-optics configuration. We also developed an appropriate recording technique to satisfy both the low-aberration condition and the Bragg condition, despite a wavelength shift between the recording and readout.
\end{abstract}

Subject terms: optical interconnections; holography; lenslet arrays.

Optical Engineering 33(2), 636-642 (February 1994).

\section{Introduction}

Planar diffractive optical elements are well suited to fulfill the needs of optical interconnections. They are efficient and can serve as focusing elements, imaging elements, beam deflectors, and fan-out elements. Almost any shape of structure can be realized. This flexibility allows complex and compact interconnections.

Two different types of diffractive optical elements exist: the holographic optical elements (HOEs) and the computergenerated elements. Modern microfabrication technology enables the realization of efficient computer-generated diffractive elements. Difficulties arise with the actual technology for the fabrication of complex structures with more than 1000 lines/mm employing blazed profiles, which is necessary for off-axis elements. Therefore, the holographic recording remains an interesting technique for elements with small features. However, the positioning precision is a problem for multifacet elements, such as lenslet arrays.

In this paper, we demonstrate how the precision of e-beam lithography and the advantage of holography can be brought together to record large lenslet arrays. Special emphasis is given to record compact lenslet arrays with short focal lengths. These elements are of interest in planar architectures. ${ }^{1}$ Near-field total internal reflection (TIR) holography ${ }^{2}$ is an appropriate technique to record such elements.

*Current affiliation: Unikeller Holding AG, CH-8306 Brüttisellen, Switzerland.

Paper 42013 received Jan. 30, 1993; revised manuscript received May 19, 1993; accepted for publication May 19, 1993.

(c) 1994 Society of Photo-Optical Instrumentation Engineers. 0091-3286/94/\$6.00
We first discuss the principle of TIR holography. Then, we show experimental results involving large lenslet arrays and clock distribution for a VLSI circuit. Finally, we present a method to record TIR holograms for readout in the IR.

\section{Near-Field TIR Holography}

\subsection{Recording Many Holographic Facets with High- Position Accuracy}

For recording HOEs with a large number of focusing facets, such as a $100 \times 100$ lenslet array, sequential recording of the facets is time consuming. In this paper, we opt for a simultaneous recording in which all object beams are recorded together. To generate the object beams we illuminate a pinhole mask with a laser beam (Fig. 1). In this manner, large arrays of spherical waves are generated simultaneously by the mask. Such pinhole masks can be fabricated as chromeon-glass masks by e-beam lithography, which assures a highposition accuracy $(\sim 0.1 \mu \mathrm{m})$ between the light sources.

To avoid intermodulations, the light from neighboring objects should not overlap in the HOE plane. The size of the pinholes in the mask influences the aperture of the object waves and also the facet size in the HOE plane. By correctly choosing the pinhole sizes, the distance between neighboring pinholes, and the distance separating mask and HOE, we can avoid overlap of the different object beams.

The pinhole size of the recording mask determines the size of the holographic facets and the size and intensity of the readout spots. When recording a mask with pinholes of different sizes, the object wave irradiance may vary considerably from one object to the other. Doubling the size of a 


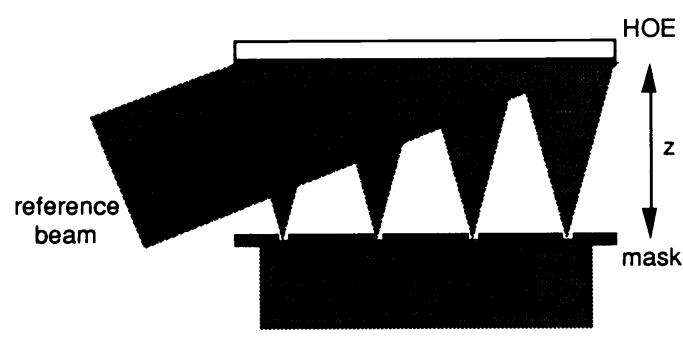

Fig. 1 Recording a HOE with a pinhole mask as the object beam generator. For short focal lengths ( $z$ small), a vignetting problem occurs for the reference wave.

pinhole will quadruple the object wave intensity and will divide by four the illumination area in the holographic plane (far-field propagation assumed). This results in an increase of 16 times the object wave irradiance and of the ratio between the object and reference beams.

The differences in pinhole size have two major consequences. First, the reference to object beam ratio cannot be optimum for all facets, because the object irradiance can vary in large proportions. Second, the size is not identical for all facets, that is, small pinholes produce large facets, which in turn collect more incident light at readout. These two disadvantages are either cumulative or compensating, depending on whether the beam ratio is optimum for large or small facets.

\subsection{TIR Holography}

The recording of HOEs with a pinhole mask as the object generator allows us to obtain very small facets and a large number of facets in one single recording. However, for transmission holographic lenses of short focal length, a vignetting problem occurs at recording because of geometry (Fig. 1). For reflection HOEs of short focal length, the same problem occurs at readout.

This vignetting problem is overcome by using near-field TIR holography, as first reported by Stetson. ${ }^{2}$ A mask placed in close proximity (typically 50 to $500 \mu \mathrm{m}$ ) to the photosensitive layer [Fig. 2(a)] is illuminated by a collimated beam and produces the object waves. These object waves interfere with the reference wave, which is fed through a prism and totally reflected at the film-air interface. This recording configuration produces three gratings: a transmission grating from interferences between the object waves and the incident reference wave, a reflection grating from the object waves and the TIR reference wave, and a Lippmann grating from the incident reference wave and the TIR reference wave.

The polarization of the recording waves is also an important parameter. The use of $p$-polarization has the advantage of producing only two of the three gratings (no Lippmann grating). On the other hand, $p$-polarization produces gratings with a smaller contrast and, thus, requires a higher exposure energy for the same permittivity modulation.

At readout, the transmission grating and the reflection grating contribute to the reconstruction of the object, but the Lippmann grating simply reflects the incident reference wave. Because of the TIR, the light not diffracted by the gratings remains in the substrate and does not affect the quality of the reconstructed object. For a reconstruction without a prism, the readout beam can be coupled into the hologram substrate by a prism or by another holographic grating, which

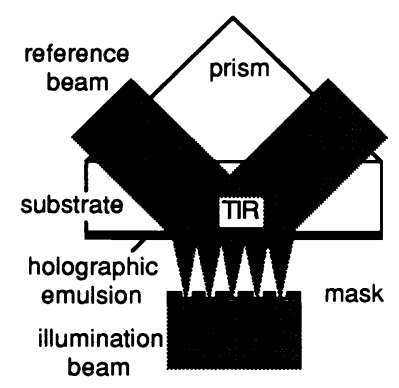

(a)

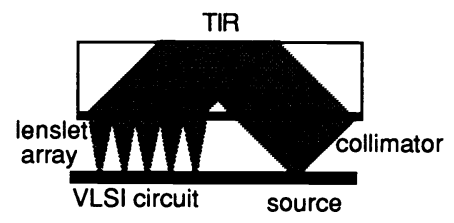

(b)

Fig. 2 (a) Recording geometry for near-field TIR holography and (b) compact readout system (doublet configuration).

can act at the same time as the collimator [Fig. 2(b)]. The grating coupler can be fabricated in the same film as the lenslet array. In this case, the collimator and lenslet array together form a compact system. Because of symmetry, this system is quite insensitive to wavelength changes.

Near-field TIR holography plays an important role in planar optics. The planar integration of free-space optical components is a very attractive concept for optical interconnections. ${ }^{1,3}$ Constraining an optical beam within a block of glass using TIR (Fig. 3) offers a number of advantages for optical system construction, namely, modularity, compactness, mechanical stability, lack of turbulence, and a reduced number of interfaces. The optical devices are placed on the surface of a glass block and are index matched to the block. This concept allows us to align all optical devices with submicron precision in the same microlithographic process.

One important optical element for the practical realization of this concept is the off-axis lenslet array of short focal length. This array permits the interfacing of planar on-axis working devices with light propagating by TIR along the substrate (see Fig. 3) and is functionally equivalent to the prism or grating coupler in 2-D integrated optics. Moreover, the lenslet allows an increase in the receiver aperture without increasing the area of the receiver itself.

\section{Experimental Realizations}

To test the performance of the near-field TIR holography, we fabricated a regular $100 \times 100$ lenslet array and also a nonregular lenslet array for clock distribution to a specially designed integrated circuit.

\subsection{High-Density Lenslet Array}

A chromium mask with $100 \times 100$ pinholes equally distributed on a surface of $1 \mathrm{~cm}^{2}$ was realized by e-beam lithography. The pinholes were $10 \times 10 \mu \mathrm{m}^{2}$ with a spacing of $100 \mu \mathrm{m}$. To avoid undesirable overlapping of the object waves, we placed the mask at about $400 \mu \mathrm{m}$ from the ho- 


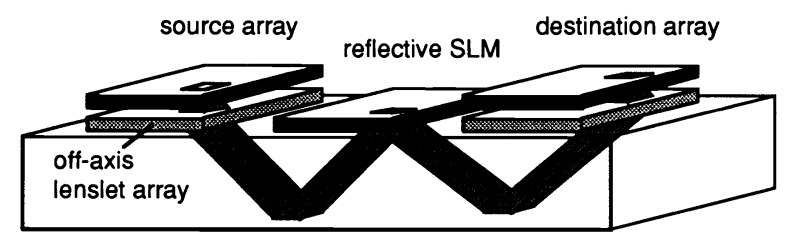

Fig. 3 Schematic diagram of a 2-D reconfigurable interconnection system.

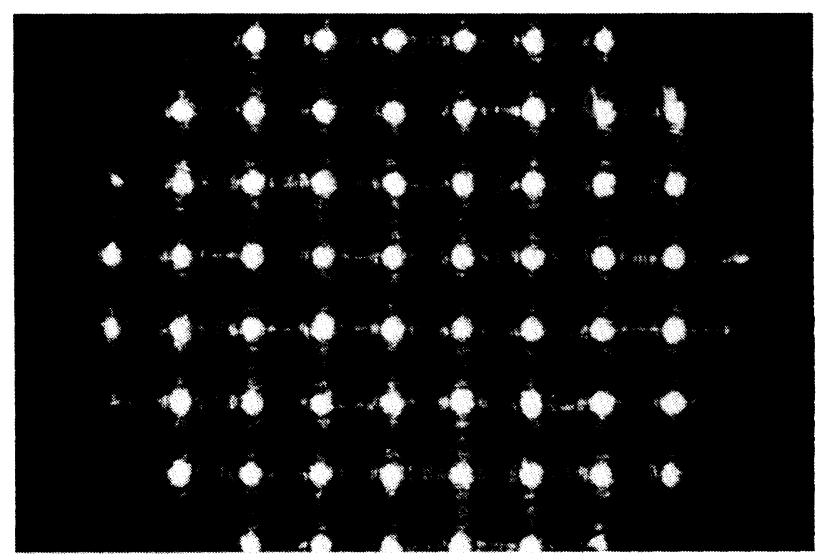

Fig. 4 Foci generated by the $100 \times 100$ lenslet array. The spot size is $10 \mu \mathrm{m}$ and the separation between two spots is $100 \mu \mathrm{m}$.

lographic emulsion and recorded it with the described nearfield TIR method.

At readout, the holographic lenslet array produces diffraction-limited spots with a focal length of $390 \mu \mathrm{m}$ and with a size of about $10 \mu \mathrm{m}$. Figure 4 shows a section of the generated spot pattern.

An application for this lenslet array has been described and realized by Collings, Prongué, and Herzig. ${ }^{4}$ The lenslet array has been used together with a liquid crystal light valve (LCLV) to form a reconfigurable interconnection system. The optical system is mounted on a glass substrate (planar optics) as shown in Fig. 5. The collimated light beam is coupled into the substrate with a prism. The substrate mode beam is reflected by the LCLV (a ferroelectric liquid crystal/amorphous silicon photoconductor sandwich) and reaches the holographic lenslet array, which focuses the light in the output plane. When a $10 \mathrm{lp} / \mathrm{mm}$ grating is written on the LCLV, then two diffracted spots appear in the focal plane of the lenslet array. These are the +1 and -1 diffracted orders of the substrate mode beam. The diffracted spots are displaced by about 10 lenslets from the central spot. Depending on the period and the orientation of the grating written on the LCLV, different locations of the lenslet array can be attained. Note that in this configuration the LCLV must show good off-axis performance and the off-axis lenslet array must have a "broad" angle acceptance (45 deg \pm 3 deg in our case).

Such programmable beam deflectors are attractive for $N \times N$ circuit switch applications. With this configuration, the link insertion loss is independent of the parallelism of the interconnection network. This is important when compared with other switching options such as the matrix-vector-

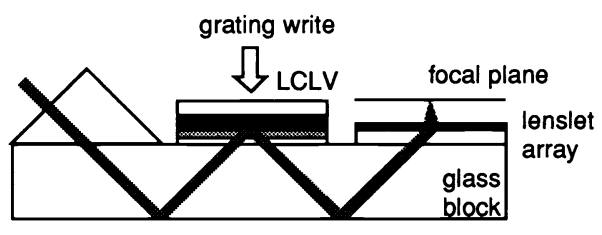

Fig. 5 Reconfigurable interconnections in planar optics.

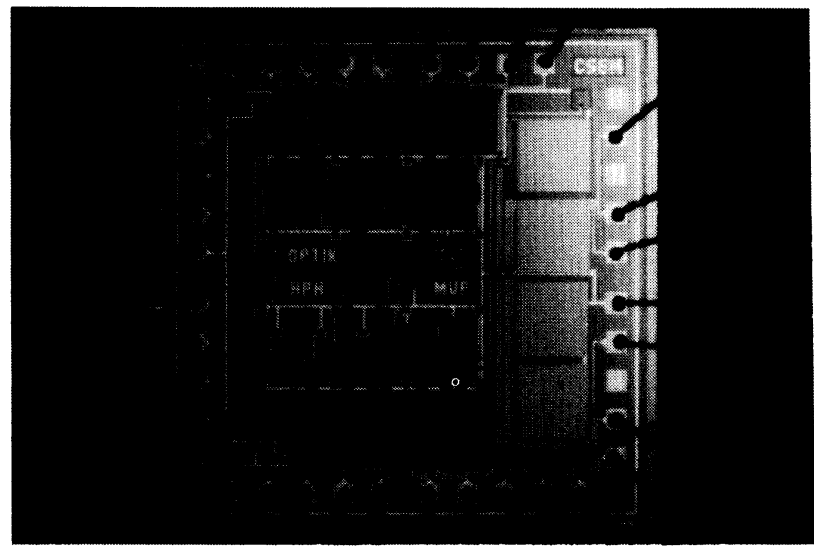

Fig. 6 VLSI chip realized by the CSEM. Its outer dimensions are $3 \times 3 \mathrm{~mm}^{2}$.

multiplier optical crossbar, in which the losses scale at least linearly with the number of links.

\subsection{Clock Distribution HOE for a VLSI Circuit}

We also applied the TIR method to fabricate a nonregular lenslet array for demonstrating clock distribution to a real VLSI circuit. For that purpose, a special test chip, shown in Fig. 6, was realized at the Centre Suisse d'Electronique et de Microtechnique (CSEM) in Neuchâtel using complementary metal oxide semiconductor (CMOS) technology (CMN20A of VLSI Technology, Inc.). Its outer dimensions are $3 \times 3 \mathrm{~mm}^{2}$. Several arrays of photodetectors with different sizes from $3 \times 3$ to $100 \times 100 \mu \mathrm{m}^{2}$ are integrated on the chip. The chip also contains transimpedance amplifiers $(R=1 \mathrm{M} \Omega)$ and output buffers to interface the photodiodes with the external electronics.

For the TIR hologram recording, a special pinhole chromium mask was fabricated by e-beam lithography. Each pinhole corresponds to a detector on the integrated circuit and acts as a source when illuminated with a laser beam. The HOE was recorded in a DuPont photopolymer by means of an argon laser at $\lambda=488 \mathrm{~nm}$.

The readout configuration is shown in Fig. 7. The readout laser beam $(\lambda=488 \mathrm{~nm})$ is coupled in the glass substrate with a deflecting HOE. After four successive TIRs, the beam reaches the focusing HOE. The hologram is placed $1 \mathrm{~mm}$ above the VLSI circuit, which is covered with a glass window.

Figure 8 shows the reconstructed spot pattern projected onto the VLSI circuit. One can see that all patterns are properly reconstructed. It has been demonstrated ${ }^{5,6}$ that the nearfield TIR method is capable of reproducing structures down to $0.4 \mu \mathrm{m}$ at $\lambda=488 \mathrm{~nm}$.

The diffraction efficiency is difficult to estimate. It varies from one facet array to the other and depends mainly on the 


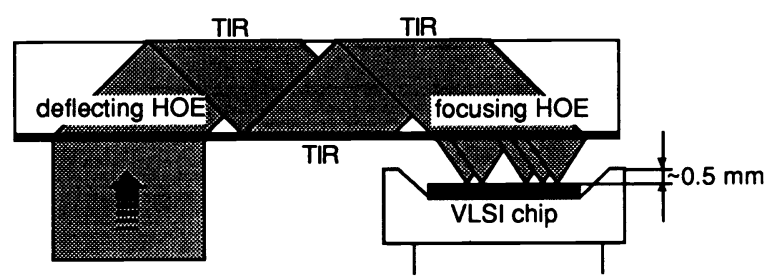

Fig. 7 Geometrical configuration at readout.

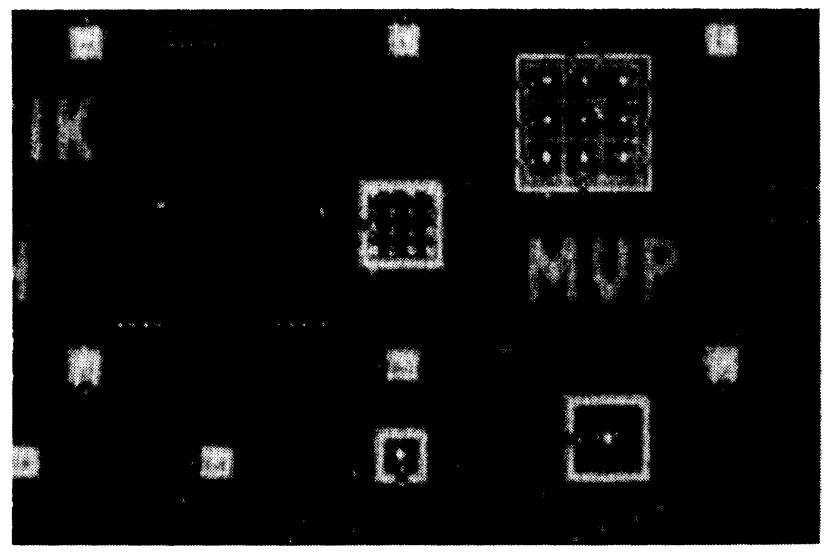

Fig. 8 Optically interconnected VLSI circuit. The spacing between the spots of the $3 \times 3$ array is $60 \mu \mathrm{m}$.

facet size and the overlap. With the range of pinhole sizes of our mask ( 3 to $10 \mu \mathrm{m}$ ), the object wave irradiance for the recording varies by a factor of $(10 / 3)^{4}=123$. Moreover, one pattern of the recording mask has pinholes with $4-\mu \mathrm{m}$ sides, separated by $8.5 \mu \mathrm{m}$. These features would require a spacing between the mask and hologram as small as $35 \mu \mathrm{m}$ to avoid an overlap of the object waves in the HOE plane. The actual spacing is much larger and the facets overlap for most of the arrayed structures. The efficiency of the hologram was only about $3.5 \%$, and we found that $50-\mathrm{nW}$ light/detector was necessary to produce a "nice" electric clock signal at the output of the VLSI circuit. Higher diffraction efficiency can be expected for optimized recording conditions (see Sec. 5).

\section{TIR Method for the Infrared}

The most attractive sources for optical interconnections are laser diodes, but most of them work at wavelengths longer than $700 \mathrm{~nm}$. Common recording materials are not sensitive to these wavelengths, but only to visible or UV light. The resulting wavelength shift between the recording and reconstruction leads to aberrations. Because the described HOEs are volume holograms, the wavelength shift may also reduce the diffraction efficiency when the Bragg condition is no longer fulfilled.

In this section, we discuss a particular design to maintain low aberrations and high diffraction efficiency in a TIR configuration. The design method was demonstrated experimentally in an earlier paper ${ }^{7}$ for a non-TIR configuration. In the following, we outline the principal results of the method and we present a numerical example for a particular case of TIR holography. A more detailed description can be found in Ref. 8.

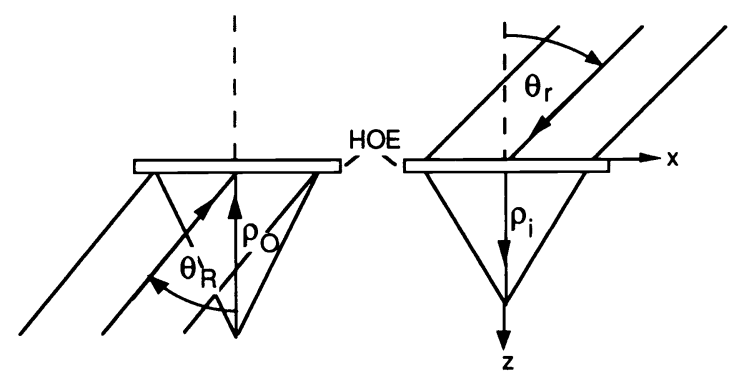

(a)

(b)

Fig. 9 (a) Recording and (b) readout of focusing HOEs.

\subsection{Low Aberrations}

To minimize the aberrations, the recording parameters, that is, the position of the sources, must be optimized. In general, simplified analytical equations are used to find an initial solution that can then be optimized by ray tracing, if necessary. In the following, we show that low aberrations are obtained by using off-axis plane waves and on-axis spherical waves in the recording and readout setup. These conditions cancel the aberrations up to third order and are simple to realize.

For the readout of a hologram, the phase $\Phi_{i}$ of the image wave is determined by

$\Phi_{i}=\Phi_{r}+m\left(\Phi_{O}-\Phi_{R}\right)$,

where $i, r, O$, and $R$ are the indices of the image, readout, object, and recording reference wave, respectively, and $m$ is the diffraction order. For the following, we consider only the first diffraction order $(m=1)$.

Our goal is to generate an on-axis spherical wave for a readout wavelength $\lambda_{r}$ that is different from the recording wavelength $\lambda_{R}$. The aberrations $\Delta \Phi(x, y)$ are then described by the difference between the image wave $\Phi_{i}(x, y)$, given by Eq. (1), and the desired spherical wave $\Phi_{d}(x, y)$, that is, by

$\Delta \Phi=\Phi_{i}-\Phi_{d}=\Phi_{r}+\Phi_{O}-\Phi_{R}-\Phi_{d}$.

We restrict ourselves to plane waves for the recording reference and for the readout wave, and to on-axis spherical waves for the object and the desired image wave. The plane waves are given by

$\Phi_{\text {plane }}=\frac{2 \pi}{\lambda} \sin \theta x$,

where $\lambda$ is the wavelength and $\theta$ the incident angle in air with respect to the hologram normal, as shown in Fig. 9. The spherical waves are described by

$\Phi_{\text {spherical }}=\frac{2 \pi}{\lambda}\left[\left(x^{2}+y^{2}+\rho^{2}\right)^{1 / 2}-\rho\right] \approx \frac{2 \pi}{\lambda}\left(\frac{r^{2}}{2 \rho}-\frac{r^{4}}{8 \rho^{3}}\right)$,

where $\rho$ is the principal radius of curvature and $r^{2}=\left(x^{2}+y^{2}\right)$. For an estimation of the aberrations, we approximated the spherical wave by a Taylor series up to fourth order.

The plane reference and readout waves are characterized by their incident angles $\theta_{R}$ and $\theta_{r}$, respectively, and the onaxis spherical object and desired image waves are charac- 
terized by their radii $\rho_{O}$ and $\rho_{d}$, respectively. From Eqs. (2) to (4), we obtain for the aberrations up to the fourth order,

$$
\begin{aligned}
\Delta \Phi= & 2 \pi\left[\frac{\sin \theta_{r} x}{\lambda_{r}}-\frac{\sin \theta_{R} x}{\lambda_{R}}+\frac{1}{\lambda_{R}}\left(\frac{r^{2}}{2 \rho_{\mathrm{O}}}-\frac{r^{4}}{8 \rho_{\mathrm{O}}^{3}}\right)\right. \\
& \left.-\frac{1}{\lambda_{r}}\left(\frac{r^{2}}{2 \rho_{d}}-\frac{r^{4}}{8 \rho_{d}^{3}}\right)\right] .
\end{aligned}
$$

The recording parameters $\theta_{R}$ and $\rho_{O}$ are free parameters to minimize the aberrations. Therefore, we choose

$\sin \theta_{R}=\frac{1}{\mu} \sin \theta_{r}$

to cancel the linear term in Eq. (5) and

$\rho_{o}=\mu \rho_{d}$

to cancel the quadratic term, where $\mu=\lambda_{r} / \lambda_{R}$ is the ratio between the readout and the recording wavelength.

From Eq. (5) the fourth-order term remains

$\Delta \Phi=\frac{2 \pi r^{4}}{8 \rho_{d}^{3} \lambda_{r}}\left(1-\frac{1}{\mu^{2}}\right)$.

In the air the numerical aperture (NA) is given by $\mathrm{NA}=$ $r / \rho_{d}$. We can now write

$\Delta \Phi=\frac{2 \pi \rho_{d}}{8 \lambda_{r}} \mathrm{NA}^{4}\left(1-\frac{1}{\mu^{2}}\right)$.

For a holographic lens with a NA $=0.2$ and focal length $\rho_{d}=1$ $\mathrm{mm}$ at the wavelength $\lambda_{r}=780 \mathrm{~nm}$, which has been recorded at $\lambda_{R}=633 \mathrm{~nm}(\mu=1.232)$, we obtain $\Delta \Phi=0.088 \times 2 \pi$. This corresponds to a wavefront error of only $\lambda / 11$.

Equation (8) shows that for a given NA, the aberrations induced by the wavelength shift are proportional to the focal length $\rho_{d}$ of the optical element. Recording with spherical and plane waves under the conditions given in Eqs. (6a) and (6b) is sufficient to obtain very small aberrations or even diffraction-limited spots for short focal lengths $\left(\rho_{d}<1 \mathrm{~mm}\right)$. For larger elements, aspherical waves obtained by computergenerated holograms (CGHs) or intermediate HOEs may be necessary to record the desired holographic phase function. ${ }^{9}$

For increasing apertures and focal lengths, higher order aberrations, more cumbersome to calculate analytically, may become dominant. They can be analyzed by ray tracing or by using scalar diffraction theory. Numerical programs using geometrical optics approximation or scalar diffraction formulas, such as the Fresnel or the Rayleigh-Sommerfeld integrals, allow us to determine the optimum distance for the image plane, the shape and size of the focused spot, and the deviation from the Bragg condition.

\subsection{Diffraction Efficiency}

Besides low aberrations of the outgoing wave, the efficiency is another important parameter of HOEs. To obtain high diffraction efficiency, we must record a volume HOE that fulfills the Bragg condition. The problem is that, if a wavelength shift occurs between the recording and reconstruction, the conditions for low aberrations and the Bragg condition are

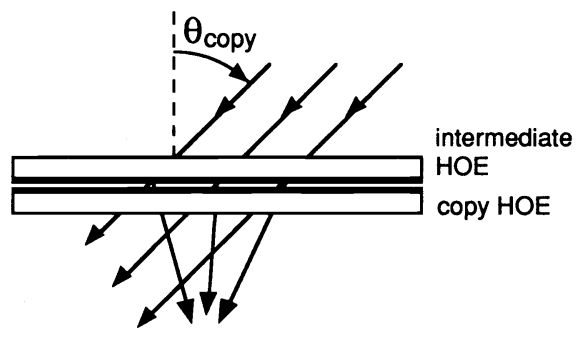

Fig. 10 Contact copy of the intermediate HOE with a new reference wave angle $\theta_{\text {copy. }}$.

not compatible in a single-step recording, without the help of special beam-shaping elements like CGHs or intermediate HOEs.

In a single-step recording, the recording beams are plane, spherical, or possibly cylindrical. The HOE can be recorded either for low aberrations or to satisfy the Bragg condition in the center of the HOE. For small NA, the spot size is determined by the diffraction limit and the geometry is chosen to satisfy the Bragg condition. For higher NAs the aberrations will certainly prevail over the diffraction limit and determine the spot size. Therefore, it will be very important to minimize the aberrations, implying that another recording method must be used if the Bragg condition is also to be satisfied.

Several methods have been proposed in the literature to satisfy the two conditions (low aberrations and high efficiency) simultaneously. They usually involve CGHs or other intermediate HOEs. ${ }^{10-13}$ These intermediate HOEs serve as aspheric wavefront generators for realizing the desired hologram structure. They must be aligned precisely in space with respect to the final hologram. This is a difficult task, especially if we require not only high optical quality (aberrations and efficiency) of the large array of recorded holograms, but also a high position accuracy. The concept of the near-field TIR holography allows alignment with submicron precision of many small holographic elements. Therefore, we propose here a copying technique, ${ }^{7}$ which preserves the $2-\mathrm{D}$ structure of the fringes with its low aberration properties and allows us to adjust the fringe tilt angle for the readout wavelength. In the first step, an intermediate HOE, optimized for low aberrations at the IR readout wavelength $\lambda_{r}$, is recorded with a visible wavelength $\lambda_{R}$. Then, in the second step (Fig. 10 ), this intermediate HOE is contact copied with another reference beam angle $\theta_{\text {copy }}$ at the wavelength $\lambda_{\text {copy }}$, which allows the adjustment of the fringe tilt of the new HOE. The contact-copying technique is insensitive to vibrations. The angle of the copying reference beam does not satisfy the Bragg condition for the intermediate HOE, thus, the intermediate HOE produces a weak object beam. This weak object beam results in a low beam ratio between the object and reference beam, that is, a low contrast of the interference pattern used to record the new HOE. The process is limited by the maximum permittivity modulation that can be achieved in the holographic layer. Now we show how to calculate the copying angle $\theta_{\text {copy }}$ to fulfill the Bragg condition at readout.

At readout, the Bragg condition ${ }^{14}$ requires

$2 \Lambda \sin \alpha_{r}=\frac{\lambda_{r}}{n}$ 
where $\Lambda$ is the fringe spacing, $\alpha_{r}$ is the angle of the readout wave in the medium with respect to the fringes, and $n$ is the refractive index (see Fig. 11). Because the copying wave is the recording reference beam of the final hologram, Eq. (9) must also be fulfilled for the copying angle $\alpha_{\text {copy }}$ at the wavelength $\lambda_{\text {copy }}$. Thus, we obtain

$\sin \alpha_{\mathrm{copy}}=\frac{\lambda_{\mathrm{copy}}}{\lambda_{r}} \sin \alpha_{r}$.

If $\delta$ is the inclination angle of the holographic fringes (Fig. $11)$, then the angles $\alpha_{\text {copy }}$ and $\alpha_{r}$ are related to $\theta_{\text {copy }}^{\prime}$ and $\theta_{r}^{\prime}$, respectively, by

$\theta_{\text {copy }}^{\prime}=\delta+\alpha_{\text {copy }}$.

$\theta_{r}^{\prime}=\delta+\alpha_{r}$.

Note that $\theta_{\text {copy }}^{\prime}, \theta_{r}^{\prime}, \delta, \alpha_{\text {copy }}$, and $\alpha_{r}$ are the angles in the medium. From Eqs. (10) and (11), we can determine the copying angle $\theta_{\text {copy }}^{\prime}$, as shown in Sec. 4.3.

With the contact-copying technique, the Bragg condition is fulfilled at the center of the facets. For high NA, the Bragg mismatch can become significant at the borders of the HOE and the diffraction efficiency will decrease. In this case, a more traditional method involving intermediate HOEs or CGHs is required.

The high spatial frequency required to obtain efficient volume holograms imposes an off-axis readout beam. Onaxis systems, which are often more convenient, can be realized with a doublet configuration consisting of a collimating HOE and a focusing multifacet HOE [see Fig. 2(b)]. The two components of the doublet HOE can be recorded with the low aberration geometry and copied to satisfy the Bragg condition. Moreover, the HOE tandem arrangement is less sensitive to wavelength variations of the source than a single element.

In summary, if we maintain the NA, the aberrations decrease with smaller system size. Therefore, for small lenslets, the spots are diffraction limited and only the Bragg condition must be satisfied. For slightly larger lenslets, we use the lowaberration geometry (plane waves or on-axis spherical waves) and we copy the element to fulfill the Bragg condition. Finally, for large lenslets, we use numerical programs to determine the optimum recording waves and we produce these waves with the aid of intermediate HOEs or CGHs.

\subsection{Particular Case of TIR hologram}

The use of efficient holographic emulsions such as a dichromated gelatin or photopolymer requires short recording wavelengths $(\leqslant 520 \mathrm{~nm})$. However, efficient semiconductor lasers work at long wavelengths $(\geqslant 780 \mathrm{~nm})$. This results in a strong wavelength shift between the recording and readout. Thus, the use of a semiconductor laser for a readout in a TIR configuration will impose a non-TIR geometry for the recording.

In that case an intermediate hologram can be first recorded in a silver halide emulsion $(n=1.6)$ at $\lambda_{R}=633 \mathrm{~nm}$ and with a recording angle $\theta_{R}^{\prime}=42 \mathrm{deg}$ in the emulsion (TIR). The HOE is then copied in an efficient holographic emulsion (dichromated gelatin or photopolymer). The copying wavelength has negligible importance as long as it is within the

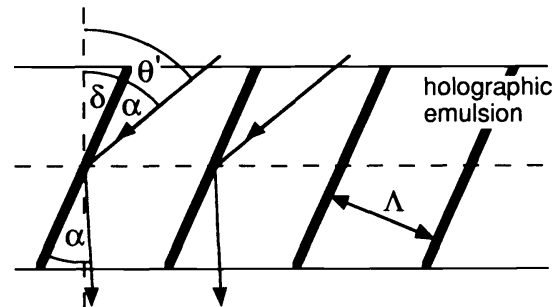

Fig. 11 Bragg diffraction of an incident beam at a thick holographic grating with slanted fringes.

sensitivity range of the holographic material. With a wavelength of $\lambda_{\text {copy }}=488 \mathrm{~nm}$, copying is realized with a prism at an angle $\theta_{\text {copy }}^{\prime}=44.6 \mathrm{deg}$ in the emulsion. The intermediate hologram and the copy are index matched to avoid undesired reflections. Replaying at $\lambda_{r}=780 \mathrm{~nm}$ will give an acceptable readout angle $\theta_{r}^{\prime}=55.5 \mathrm{deg}$ in the emulsion (TIR). In the copying process, the Bragg angle can also be readjusted. The angles have been calculated from Eqs. (10) and (11). Note that the readout angle $\theta_{r}^{\prime}=55.5 \mathrm{deg}$ generates an on-axis image wave, that is, $\theta_{i}^{\prime}=0 \mathrm{deg}$. The inclination angle of the holographic fringes then becomes $\delta=\theta_{r}^{\prime} / 2=27.75 \mathrm{deg}$ (see Fig. 11).

\section{Discussion}

Compared with computer-generated lenslet arrays, the holographic method (recording by interference) has the advantage that holographic materials with very high resolution can be used. The recording of gratings with more than 2000 lines/ $\mathrm{mm}$ is standard for materials such as DCG, photopolymers, and photoresists. The near-field TIR holography is very powerful to record large numbers of regularly spaced facets of short focal lengths. The size of the pinholes and the distance between the mask and HOE must be appropriately chosen to avoid overlapping of the diffraction patterns, which would cause intermodulations.

The object beam arriving on a facet is not uniform ("sinc" shape) and, therefore, the facet boundaries are not well defined and the fringe contrast is much lower in the boundary zones. This inevitably limits the diffraction efficiency of the facets.

The presence of three holograms in TIR holography can also limit the diffraction efficiency. The relative efficiencies of the three holograms are not known. However, Ehbets, Herzig, and Dändliker ${ }^{15}$ have shown recently that high efficiency should be possible with TIR holography. They have obtained $80 \%$ efficiency with a photopolymer holographic layer.

For recording and readout with different wavelengths, we have shown that aberrations up to a third order can be canceled. Second- and third-order aberrations are eliminated by using off-axis plane waves and on-axis spherical waves in the recording and readout setup. But it is not possible to cancel simultaneously all aberrations for the entire HOE. However, we can minimize the aberrations (all orders together) by optimizing numerically the recording geometry with a ray-tracing program.

Both the Bragg condition and the condition for low aberrations can be fulfilled simultaneously using the contactcopying method. However, both conditions are only fulfilled rigorously near the center of the HOE. 


\section{Conclusion}

The holographic recording of diffractive optical elements is of interest for realizing highly off-axis elements such as those needed in planar architectures, which require high spatial frequencies $(>1000$ lines $/ \mathrm{mm})$. Critical items in fabricating such compact optical systems with small holographic elements are the geometrical restrictions to record the holograms (beam obstructions), the accurate position between the elements, the aberrations of the reconstructed beams, and the diffraction efficiency.

The vignetting problem is overcome by applying nearfield TIR holography. The object waves are generated by an e-beam mask. This concept allows alignment with submicron precision of all optical devices realized in the same microlithographic process. With this method, a holographic lenslet array with 10,000 interconnection points of $390-\mu \mathrm{m}$ focal length and diffraction-limited spot size of $10 \mu \mathrm{m}$ has been fabricated. The TIR method has also been used to realize a clock distribution HOE for a specially designed VLSI circuit.

Furthermore, we have investigated how low aberrations and high diffraction efficiency can be obtained with the nearfield TIR technique despite a wavelength shift between the recording and readout. For a given aperture, the geometrical aberrations are scaled with the size of the element. Therefore, for small lenslets, the spots are diffraction limited and we must fulfill only the Bragg condition, which is necessary for high diffraction efficiency. For slightly larger lenslets, we use the low aberration geometry (off-axis plane waves and on-axis spherical waves) and we copy the element to fulfill the Bragg condition. Finally, for large lenslets, we use numerical programs to determine the optimum recording waves and we produce these waves with the aid of intermediate HOEs or CGHs.

\section{Acknowledgment}

The authors gratefully acknowledge the helpful advice of Prof. R. Dändliker.

\section{References}

1. J. Jahns and A. Huang, "Planar integration of free-space optical components," Appl. Opt. 28(9), 1602-1605 (1989).

2. K. Stetson, "Holography with total internally reflected light," Appl. Phys. Lett. 11(7), 225-226 (1967).

3. K.-H. Brenner and F. Sauer, "Diffractive-reflective optical interconnects," Appl. Opt. 27(20), 4251-4254 (1988).

4. N. Collings, D. Prongué, and H. P. Herzig, "Two-dimensional reconfigurable interconnect in a planar optics configuration," in OSA Proceedings on Photonic Switching, H. S. Hinton and J. W. Goodman, Eds., Vol. 8, 81-84, OSA, Washington, DC (1991).

5. R. Dändliker and J. Brook, "Holographic photolithography for submicron VLSI structures," in Holographic Systems, Components and
Applications, Conference Publication Number 311, 127-132, Institution of Electrical Engineers, London (1989).

6. R. T. Chen, L. Sadovnik, T. M. Aye, and T. Jannson, "Submicron lithography using lensless high-efficiency holographic systems," Opt. Lett. 15, 868-871 (1990).

7. D. Prongué and H. P. Herzig, "Design and fabrication of HOE for clock distribution in integrated circuits," in Holographic Systems, Components and Applications, Conference Publication Number 311, 204-208, IEE, London (1989).

8. D. Prongué, "'Diffractive optical elements for interconnections," $\mathrm{PhD}$ Thesis, University of Neuchâtel, Switzerland (1992).

9. H. P. Herzig and R. Dändliker, "Holographic optical elements for use with semiconductor lasers," in International Trends in Optics, J. W. Goodman, Ed., pp. 57-75, Academic Press, New York (1991).

10. H. P. Herzig, "Holographic optical elements (HOE) for semiconductor lasers," Opt. Commun. 58(3), 144-148 (1986).

11. H. Buczek and J. M. Teijido, "Application of electron-beam lithography at CSEM for fabricating computer-generated holograms," Proc. SPIE 884, 46-51 (1988)

12. M. Assenheimer, Y. Amitai, and A. A. Friesem, "Recursive design for an efficient HOE with different recording and readout wavelength," Appl. Opt. 27(28), 4747-4752 (1988).

13. Y. Amitai and J. W. Goodman, "Design of substrate-mode holographic interconnects with different recording and readout wavelengths," Appl. Opt. 30(17), 2376-2381 (1991)

14. A. Yariv, Optical Electronics, p. 387, Holt-Saunders International Editions, New York (1985).

15. P. Ehbets, H. P. Herzig, and R. Dändliker, "TIR holography analyzed by coupled wave theory," Opt. Commun. 89, 5-11 (1992).

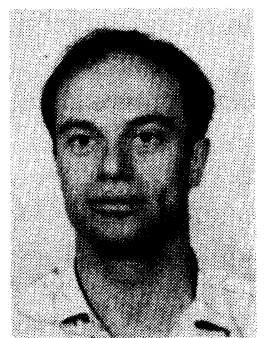

Damien Prongue received the diploma in electrical engineering from the Swiss Engineering College in Le Locle, Switzerland in 1981 and the diploma in physical electronics from the University of Neuchâtel, Switzerland, in 1986. He then joined the Applied Optics Group at the Institute of Microtechnology of the University of Neuchâtel, Switzerland, as a graduate research assistant working on holographic optical elements. In 1992, he received the PhD degree in optics from the University of Neuchâtel for his work on diffractive optical elements for interconnections. He is now with Unikeller Holding AG, Brüttisellen, Switzerland.

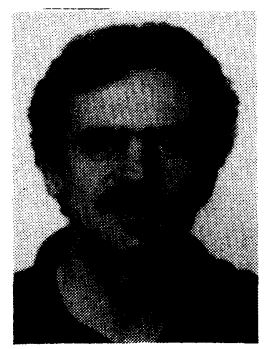

Hans Peter Herzig received the diploma in physics from the Swiss Federal Institute of Technology in Zürich (ETHZ), Switzerland, in 1978. From 1978 to 1982 , he was a scientist in the Optics Development Department of Kern in Aarau, Switzerland where he worked in lens design and optical testing. In 1983, he joined the Applied Optics Group at the Institute of Microtechnology of the University of Neuchâtel, Switzerland, as a graduate research assistant working in the field of holographic optical elements, especially scanning elements. In 1987, he received the PhD degree in optics from the University of Neuchâtel. His current research deals with diffractive optical elements, optical interconnections, and beam shaping. He is a member of the Optical Society of America, of the European Optical Society, and of the Swiss Optical Society. 